\begin{tabular}{|c|c|c|}
\hline & Int.J.Curr.Microbiol.App.Sci (2021) 10(11): 200-206 & \\
\hline & $\begin{array}{l}\text { International Journal of Current Microbiology and Applied Sciences } \\
\text { ISSN: 2319-7706 Volume } 10 \text { Number } \mathbf{1 1} \mathbf{( 2 0 2 1 )} \\
\text { Journal homepage: http://www.ijcmas.com }\end{array}$ & $\$$ \\
\hline $\begin{array}{l}\text { EXCELLENT } \\
\text { PUBLISHERS }\end{array}$ & & \\
\hline
\end{tabular}

Case Study

https://doi.org/10.20546/ijcmas.2021.1011.023

\title{
A Rare Case Report of Splenic Abscess Caused by Burkholderia pseudomallei Progressing to Disseminated Melioidosis
}

\author{
Lakshmi Shanmugam ${ }^{1}$, Priyadarshi Ketan ${ }^{1}$, Stanley Pheba ${ }^{1}$, Monika Sivaradjy ${ }^{2}$, \\ S. Balamurugan ${ }^{3}$, Elamurugan Thirthar Palanivelu ${ }^{3}$ and Sastry Apurba Sankar ${ }^{\text {** }}$
}

${ }^{1}$ Department of Microbiology, Jawaharlal Institute of Postgraduate Medical Education and Research, Puducherry - 605006, India

${ }^{2}$ Department of Microbiology, Jawaharlal Institute of Postgraduate Medical Education and Research, Karaikal, India

${ }^{3}$ Department of General Surgery, Jawaharlal Institute of Postgraduate Medical Education and Research, Puducherry - 605006, India

*Corresponding author

\begin{tabular}{|c|}
\hline Keywords \\
\hline $\begin{array}{l}\text { Splenic abscess, } \\
\text { Burkholderia } \\
\text { pseudomallei }\end{array}$ \\
\hline Article Info \\
\hline $\begin{array}{l}\text { Received: } \\
\text { 10 October } 2021 \\
\text { Accepted: } \\
05 \text { November } 2021 \\
\text { Available Online: } \\
10 \text { November } 2021\end{array}$ \\
\hline
\end{tabular}

A B S T R A C T

Splenic abscess is a rare but potentially life threatening condition which can be less frequently caused by gram negative saprophytes, Burkholderia pseudomallei. Because of the challenges faced in the identification of this organism, it is often under diagnosed or misdiagnosed. Here, we report a case of splenic abscess eventually leading to disseminated melioidosis caused by Burkholderia pseudomallei in a 40 year old diabetic male from a tertiary-care hospital in southern India, who presented with fever and pain over left upper abdomen.

\section{Introduction}

Splenic abscess is a rare clinical entity estimated to have a frequency of about less than $0.7 \%$, based on autopsy studies. If left untreated, it is associated with a high rate of mortality. ${ }^{[1]}$ The most common organisms known to cause splenic abscess are Streptococcus spp. and Staphylococcus aureus, but Escherichia coli, Proteus mirabilis, Klebsiella pneumoniae, Salmonella species, Enterococcus spp, Pseudomonas species as well as fungi like Candida, Aspergillus, and Cryptococcus species can also be the other culprits. A combination of computed tomography (CT), abdominal ultrasonography (US) and clinical features found to be helpful in early diagnosis. The management of the condition includes medical therapy with broad spectrum of antibiotics, CT-guided percutaneous aspiration, and splenectomy. Here we are discussing a case of 
multiple splenic abscess in a $40 \mathrm{yr}$ old male caused by Burkholderia pseudomallei which later progressed to cause bloodstream infection resulting in disseminated melioidosis.

\section{Case Presentation}

A $40 \mathrm{yr}$ old male farmer hailing from Vadalur, a small district in South India presented to our hospital with high grade fever and pain over left upper abdomen since 4 days. Pain was acute in onset, progressive, dull continuous, non radiating, not relieved on medications. It was associated with multiple episodes of high grade fever and chills. The patient did not have any complaints of vomiting, abdominal distension, jaundice, altered bowel habits, cough, reduced urine output or gastrointestinal bleed. This person is a known diabetic for 5 years on irregular medications, chronic alcoholic for 10 years but not a smoker or intravenous drug abuser. There is an history of undocumented liver and splenic abscess 1 year back for which he has taken some native medications, the details of which is he is not aware of. There was no history of any previous surgery. He was initially evaluated in Medicine OPD and was referred to the Department of General Surgery in view of suspected rupture of splenic abscess.

Clinical examination revealed that the patient was conscious, oriented, moderately built and nourished. His vitals were normal with good hydration status. He was found to have icterus but there were no signs of pallor, pedal edema, lymphadenopathy.

Abdomen was found to be soft, tender and distended with localised guarding present in the left hypochondriac region. Liver was enlarged upto $5 \mathrm{~cm}$ below right costal margin and spleen tip was palpable with normal bowel sounds.
Ultrasonography (USG) of the abdomen showed hepatosplenomegaly with a spleen size of $13.3 \mathrm{~cm}$, mildly enlarged with rounded margins, multiple hypoechoic lesions were seen in the region of the mid pole, with the largest lesion measuring around $1.5 \times 1.5 \mathrm{~cm}$ without any obvious internal vascularity.

There was no free fluid in abdomen. The liver size was $16.7 \mathrm{~cm}$ with homogeneously increased echoes with mild gallbladder wall edema. Pancreas was found to be normal.

Contrast enhanced computed tomography (CECT) Abdomen and Thorax showed hepatomegaly with multiple small hypodense lesions in spleen with minimal perisplenic collection and left sub diaphragmatic collection near upper pole. The collection was closely abutting the tail of pancreas and anteriorly into lesser sac. Mild left pleural effusion was noted with multiple enlarged para-aortic, paracaval, aortocaval lymph nodes (Figures 1 and 2).Two Dimensional (2D) Echocardiography showed a normal study.

Paired blood culture, comprising of $9 \mathrm{~mL}$ of blood collected from the peripheral line were loaded into an automated blood culture bottle of the Virtuo BacT/ALERT 3D system, were sent on the day of admission which turned out to be sterile after 5 days of aerobic incubation.

Laboratory investigations showed dearranged liver profile with hyperbilurubinemia (total bilurubin $5.95 \mathrm{mg} / \mathrm{dL}$, Direct bilurubin 3.88 $\mathrm{mg} / \mathrm{dL}$ ) hypoalbuminemia and elevated levels of aspartate aminotransferase (AST $74 \mathrm{IU} / \mathrm{L}$ ), alkaline phosphatase (ALP $164 \mathrm{IU} / \mathrm{L}$ ) and Gamma-glutamyl transferase (GGT 112 $\mathrm{IU} / \mathrm{L})$. Also found to have moderate anemia with a hemoglobin level of $7 \mathrm{gm} / \mathrm{dl}$. He also had uncontrolled hyperglycemia with random blood glucose levels of $275 \mathrm{mg} / \mathrm{dl}$ with poor glycemic control with a HbA1c of $14.4 \%$.

The patient was diagnosed with ruptured 
splenic abscess with septic shock and was empirically started on Inj.vancomycin $1 \mathrm{gm}$ twice daily and Inj meropenem $1 \mathrm{gm}$ thrice daily. He was planned for emergency splenectomy since he had multiple foci of pus pockets in spleen and was known case of diabetic. Open splenectomy with primary repair of colonic fistula was carried out.

Intra operative findings were enlarged liver with fatty changes. Mild splenomegaly was present with multiple partially organised abscesses in inferior, posterior, superolateral and medial aspects of spleen. Dense adhesions were present between abscess cavities and stomach, colon and diaphragm. Small, around $0.5 \mathrm{~cm}$ fistulous communication of inferior abscess cavity with splenic flexure of colon. Dense vascular adhesions were present between spleen and retro peritoneum and diaphragm. Fistula edges freshened and primarily repaired in 2 layers. Splenic hilum was taken down with vascular stapler. There was no free fluid in the abdominal cavity.

Intra operative pus was sent for culture and sensitivity. Direct gram staining was performed which revealed the presence of pus cells and gram negative bacilli. The pus sample was cultured onto 5\% sheep blood agar and MacConkey agar and incubated at $37^{\circ} \mathrm{C}$ for 16-18 hours. Blood agar showed smooth, mucoid to dry wrinkled colonies (Figure 3) and MacConkey agar showed flat non lactose fermenting colonies with metallic sheen (Figure 4).Culture smear was done which showed small, gram negative bacilli with bipolar staining giving a safety pin appearance (Figure 5).Colonies were identified by VITEK® 2 system (Biomeriux) as Burkholderia pseudomallei. Minimum inhibitory concentration (MIC) were determined by automated VITEK® 2 system and interpreted using Clinical and Laboratory Standards Institute breakpoints (CLSI M45A2) and the isolate was found to be sensitive to imipenem ( $\mathrm{MIC} \leq 4 \mu \mathrm{g} / \mathrm{ml})$ and resistant to ceftazidime (MIC $32 \mu \mathrm{g} / \mathrm{ml}$ ) and cotrimoxazole (MIC 4/76 $\mu \mathrm{g} / \mathrm{ml}$ ) intermediate to levofloxacin (MIC $4 \mu \mathrm{g} / \mathrm{ml}$ ) and minocycline (MIC $8 \mu \mathrm{g} / \mathrm{ml}) .{ }^{[2]}$

Fever spikes were there on post-operative day 5 for which paired blood culture were sent in BacT/Alert bottles which were loaded into BACT/ALERT 3D system which flagged positive in 29 hours and 32 hours. From the blood culture bottles, gram stain was performed which showed small, gram negative bacilli with bipolar staining. Further, it was subcultured onto 5\% sheep blood agar and MacConkey agar. Similar type of colonies were observed in both the culture plates and were subsequently identified as Burkholderia pseudomallei and MIC were determined and interpreted using CLSI M45-A2 breakpoints and was found to be sensitive to ceftazidime (MIC $4 \mu \mathrm{g} / \mathrm{ml}$ ), cotrimoxazole (MIC 2/38 $\mu \mathrm{g} / \mathrm{ml}$ ) and imipenem (MIC $4 \mu \mathrm{g} / \mathrm{ml}$ ).

The patient was continued on Inj. meropenem for 4 weeks till the fever spikes settled. Glycemic control was achieved with injection insulin. One unit of packed cells was transfused. Blood culture was repeated after 4 weeks which turned out to be sterile. Post splenectomy vaccination was given. The patient improved clinically and was discharged with oral cotrimoxazole for 3 months for eradication therapy.

\section{Results and Discussion}

Splenic abscess, though a rare entity, is a potentially life threatening condition. The most common mode of infection is hematogenous seeding of bacteria from a secondary site (e.g., heart, lung, urinary tract). Other well-known predisposing conditions are splenic trauma and hemoglobinopathies. The most vulnerable population include those with immunodeficiency, neoplasia, trauma, 
metastatic infection, splenic infarct or diabetes. The most common presentation of splenic abscess are fever, abdominal pain, nausea and vomiting. Solitary splenic lesions is uncommon. Multiple splenic abscesses are still a relatively rare finding encountered predominantly in immunocompromised patients and in those with underlying malignancies. $^{[3]}$

Although splenic abscess can be caused by a wide range of microbes, rarely it can also be caused by a gram negative saprophytic organism, Burkholderia pseudomallei, resulting in Melioidosis. It was in April 1911,when Alfred whitemore and C. S. Krishnaswami first recognized it in Rangoon, Burma, hence it was called the Whitmore's disease. ${ }^{[4]}$ This organism is commonly found in contaminated water, soil, rice paddies, and ponds throughout the endemic regions.

A modeling study conducted in 2016 estimated that there were $\sim 165,000$ cases of melioidosis in humans per year worldwide, of which 89,000 (54\%) were estimated to be fatal. ${ }^{[5]}$ In endemic areas, the mortality rate ranges from $19 \%$ to $36 \%$. Melioidosis is endemic in South and East Asia, Northern Australia, the Indian subcontinent, and areas of South America. ${ }^{[6]}$ Melioidosis is a disease of all age groups. Prospective studies conducted in Australia and Thailand showed the median age, the median age of patients with melioidosis were 50 years, with $5-10 \%$ being less than $<15$ years. ${ }^{[7]}$ Predisposing conditions for melioidosis includes diabetes mellitus, chronic lung and renal disease and other immunocompromised conditions. Curie et al., found that the individuals with diabetes mellitus have a 12-fold higher risk of melioidosis after adjustment for age, sex and other risk factors. Aneesh et al., have studied that the common risk factors were diabetes $(83.9 \%)$ and alcohol abuse $(58.1 \%){ }^{[8]}$

People can acquire melioidosis through contact with contaminated soil and surface waters by percutaneous inoculation, aerosol inhalation, or the ingestion of contaminated water or food. Various factors like inoculating dose, mode of infection, host risk factors, and probably differential virulence of infecting $B$. pseudomallei strains can have their influence over the incubation period. Onset of melioidosis within 24 hours has been seen in presumed aspiration after near drowning and, in some cases, after severe weather events.

Clinical syndromes include subclinical infections, asymptomatic or minor localized abscesses, severe pneumonia, and fulminant sepsis. Septicemia and community acquired pneumonia remains as the most frequent presentation. Only a few cases of splenic abscess due to melioidosis have been reported, even in endemic areas.

Burkholderia pseudomallei is an aerobic, nonspore-forming, straight, slender, gram negative bacilli with cells that range from 1 to $5 \mu \mathrm{m}$ long and 0.5 to $1 \mu \mathrm{m}$ wide motile. ${ }^{[9]}$

B. pseudomallei being a facultative intracellular organism, invades and replicates inside polymorphonuclear leukocytes, macrophages and some epithelial cell lines.

Lipopolysaccharide [LPS] confers resistance to human serum and the capsular polysaccharide is responsible for its intracellular survival. Both these appear to be crucial in the pathogenesis of melioidosis. Type III and type VI secretion systems are also found to be important in cell invasion and intracellular survival. 
Fig.1 A) Coronal view of CECT Abdomen showing hepatomegaly with multiple small hypodense lesions in spleen (arrows) with minimal perisplenic collection.

B) $5 \%$ sheep blood agar showing smooth, mucoid to dry wrinkled colonies.

C) MacConkey agar showing flat non lactose fermenting colonies with metallic sheen.

D) Gram staining of colony showing gram negative bacilli with safety pin appearance

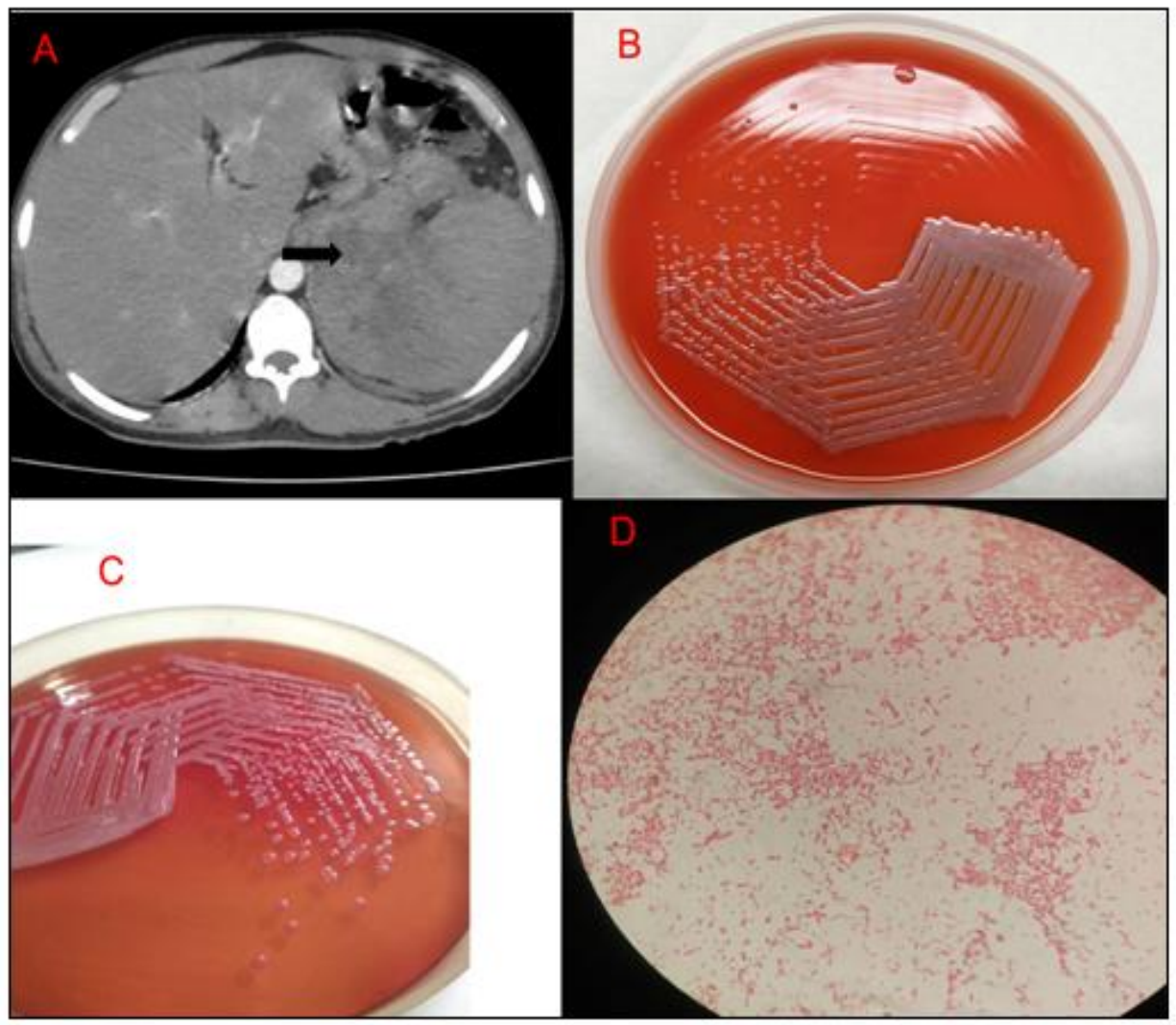

Quorum sensing plays an important role in cell invasion, cytotoxicity, and antimicrobial resistance. Other weapons for virulence include flagella, type IV pili and other adhesins, a siderophore, and secreted proteins, such as hemolysin, lipases, and proteases. Burkholderia lethal factor-1 is similar to Escherichia coli cytotoxic necrotizing factor-1 and interferes with initiation of translation, leading to alteration of the actin cytoskeleton and ultimately cell death. ${ }^{[9]}$

Definitive diagnosis of melioidosis requires the isolation and identification of $B$. pseudomallei from clinical specimens. It is a gram negative bacilli with safety pin appearance which is because of its bipolar staining. This organism readily grows in commercially available blood culture media, but the most common problem in nonendemic locations is that it can be misidentified as a Pseudomonas as the dry, wrinkled colonies morphologically resembles Pseudomonas stutzeri or other Burkholderia species, especially because some commercial identification systems are poor at identifying B. pseudomallei. The rate of successful culture is increased if sputum, throat swabs, ulcer or skin lesion swabs, and rectal swabs are placed into Ashdown's medium, a gentamicincontaining liquid transport broth that results in the selective growth of $B$. pseudomallei, or 
plated onto Ashdown's agar or Burkholderia pseudomallei selective agar (BPSA). ${ }^{[9]}$

Matrix-Assisted Laser Desorption/Ionization Time of Flight Mass Spectrometry (VITEK® MS) (MALDI TOF-MS) has been used for rapid identification of $B$. pseudomallei in clinical laboratories. However, existing databases do not contain enough spectra for the species identification of $B$. pseudomalle $i$, which is uncommonly encountered outside endemic areas. Optimization of the databases by adding more reference spectra for $B$. pseudomallei and related species is critical to enable accurate identification, especially in countries where melioidosis is prevalent, and should be adopted in standard MALDI-TOF MS libraries used in clinical laboratories. Lowe and colleagues compared the accuracy of four systems, the manual APE 20NE and 20E, and the automated Vitek 1 and 2 systems (bioMérieux Inc. Hazelwood, MO) for their ability to correctly identify 103 strains of $B$. pseudomallei. The API 20NE, API 20E, and Vitek 1 systems gave the correct identification in $98 \%, 99 \%$, and $99 \%$ of the isolates, respectively. The Vitek 2 failed to identify a large number of the B. pseudomallei strains largely because of differences in the biochemical reactions achieved compared to expected values in the database. ${ }^{[10]}$ Immunochromatographic test (ICT) for antigen detection targeting capsular polysaccharides and $h c p l$ are also available for diagnosis.

B. pseudomallei is intrinsically resistant to penicillin, ampicillin, first and second generation cephalosporins, gentamicin, tobramycin, and streptomycin. $B$. pseudomalle $i$ is susceptible to various $\beta$ lactam antibiotics, especially ceftazidime, imipenem, meropenem, piperacillin, amoxicillin-clavulanate, ceftriaxone, and cefotaxime, with various degrees of bactericidal activity. Treatment comprises of intensive and eradication therapy. Initial intensive therapy can be carried out with ceftazidime $(50 \mathrm{mg} / \mathrm{kg}$, up to $2 \mathrm{~g})$ every 6 hourly or meropenem ( $25 \mathrm{mg} / \mathrm{kg}$, up to $1 \mathrm{~g}$ ) every 8 hourly or Imipenem $(25 \mathrm{mg} / \mathrm{kg}$, up to $1 \mathrm{~g}$ ) every 6 hourly for a minimum of 10-14 days. Any one of the three may be combined with co-trimoxazole $(6 / 30 \mathrm{mg} / \mathrm{kg}$, up to $320 / 1600 \mathrm{mg}$ ) every 12 hourly (recommended for neurologic, cutaneous, bone, joint, and prostatic melioidosis) followed by eradication therapy with co-trimoxazole $(6 / 30 \mathrm{mg} / \mathrm{kg}$ up to $320 / 1600 \mathrm{mg}$ ) every 12 hourly for a minimum of 3 months. ${ }^{[9]}$

Even though Burholderia pseudomallei rarely causes splenic abscess, it could be highly fatal if left untreated or not promptly treated. This case was managed efficiently because of the strong clinical suspicion as well as with the help of timely microbiological investigations and diagnosis. This case suggests that there is a need for improved microbiological diagnostic modalities that could aid in the early diagnosis thereby facilitating prompt treatment of the patient.

\section{References}

1. Robert S. Ohgami M D, PhD,... Daniel A. Arber MD, in Hematopathology (Third Edition), 2018

2. M45-A2: Methods for Antimicrobial Dilution and Disk Susceptibility Testing of Infrequently Isolated or Fastidious Bacteria; Approved Guideline - Second Edition. : 100 .

3. Saber A. Multiple splenic abscesses in a rather healthy woman: a case report. Cases Journal. 2009 Dec;2(1):1-6.

4. Whitemore A, Krishnaswami C S: An account of the discovery of hitherto undescribed infective disease occurring among the population of Rangoon. Ind Med Gaz. 1912, 47:262-267

5. Limmathurotsakul D, Golding N, Dance D 
A, Messina J P, Pigott D M, Moyes C L, Rolim D B, Bertherat E, Day N P, Peacock S J, Hay S I. Predicted global distribution of Burkholderia pseudomallei and burden of melioidosis. Nature microbiology. 2016 Jan 11;1(1):1-5.

6. Kandhasamy S C, Elamurugan T P, Naik D, Rohith G, Ramakrishnaiah V P. Systemic Melioidosis with Ruptured Splenic Abscess. Cureus. 2020 May;12(5).

7. McLeod C et al., Clinical presentation and medical management of melioidosis in children: a 24- year prospective study in the Northern Territory of Australia and review of the literature. Clin. Infect. Dis 60,
21-26 (2015).

8. Basheer A, Iqbal N, Kanungo R, Kandasamy R. Melioidosis: distinctive clinico-epidemiological characteristics in southern India. Tropical Doctor. $2020 \mathrm{Jul}$ 29:0049475520943698

9. Bennett J E, Dolin R, Blaser M J. Mandell, Douglas, and Bennett's Principles and Practice of Infectious Diseases E-Book. Elsevier Health Sciences; 2019.

10. Lowe P, Engler C, Norton R. Comparison of automated and nonautomated systems for identification of Burkholderia pseudomallei. J Clin Microbiol 2002;40:4625-4627.

\section{How to cite this article:}

Lakshmi Shanmugam, Priyadarshi Ketan, Stanley Pheba, Monika Sivaradjy, S. Balamurugan, Elamurugan Thirthar Palanivelu and Sastry Apurba Sankar. 2021. A Rare Case Report of Splenic Abscess Caused by Burkholderia pseudomallei Progressing to Disseminated Melioidosis. Int.J.Curr.Microbiol.App.Sci. 10(11): 200-206.

doi: https://doi.org/10.20546/ijcmas.2021.1011.023 